\title{
Synthesis of 86 species of 1,5-diaryl-3-oxo- 1,4-pentadienes analogs of curcumin can yield a good lead in vivo
}

\author{
Chieko Kudo ${ }^{1,2}$, Hiroyuki Yamakoshi ${ }^{3}$, Atsuko Sato ${ }^{1}$, Hiroshi Nanjo ${ }^{4}$, Hisatsugu Ohori ${ }^{1,2}$, Chikashi Ishioka ${ }^{1,2}$, \\ Yoshiharu Iwabuchi ${ }^{3^{*}}$ and Hiroyuki Shibata ${ }^{1,5^{*}}$
}

\begin{abstract}
Background: Curcumin is known to possess many anti-tumor properties such as inhibition of tumor growth and induction of apotosis. However, limited bioavailability of curcumin prevents its clinical application. A synthesized curcumin analog, 1,5-diaryl-3-oxo-1,4-pentadiene such as GO-Y030, has the improved anti-tumor potential in vitro as well as in mouse model of colorectal carcinogenesis.

Results: These compounds were divided into two groups; one is the higher anti-proliferative group, in which 79.7\% of 1,5-diaryl-3-oxo-1,4-pentadienes were clustered. One of the 1,5-diaryl-3-oxo-1,4-pentadiene analogs, GOY078 has the most enhanced growth inhibition, and its solubility was improved, compared with curcumin. GOY078 inhibits NF- $\kappa$ B transactivation, as well as expression of TP53 and DR5 more effectively than curcumin. In a mouse model, GO-Y078 presented 1.4 fold more survival elongation that was not achieved by curcumin and GOY030.
\end{abstract}

Conclusions: The 1,5-diaryl-3-oxo-1,4-pentadiene analogs can yield good lead compounds for cancer chemotherapy, to overcome low bioavailability of curcumin.

\section{Background}

Naturally derived products are the most valuable source for drugs and their lead compounds. Approximately $74 \%$ of anticancer drugs are either natural products or their derivatives [1]. Curcumin, 1,7-bis(4-hydroxy-3methoxyphenyl)-1,6-heptadiene-3,5-dione, is a dietary constituent of turmeric. It is well known for its ability to suppress tumor growth [2]. The mechanisms of its tumor suppression have been examined at the molecular level. Curcumin is known to interfere with the transactivation of nuclear factor $-\kappa \mathrm{B}(\mathrm{NF}-\kappa \mathrm{B})$ [3], activator protein $1[4]$, and $\beta$-catenin [5] thus resulting in the negative regulation of various oncogenes such as $c-M y c$, cyclin D1, Bcl-2, and Bcl-XL. Curcumin arrests the cell

\footnotetext{
* Correspondence: iwabuchi@mail.pharm.tohoku.ac.jp; hiroyuki@med.akita-u. ac.jp

'Dept. Clinical Oncology, Institute of Development, Aging, and Cancer, Tohoku University, Seiryo-cho 4-1, Aoba-ku, Sendai, Japan ${ }^{3}$ Dept. Organic Chemistry, Graduate School of Pharmaceutical Science, Tohoku University, Aoba 6-3, Aramaki, Aoba-ku, Sendai, Japan Full list of author information is available at the end of the article
}

cycle at $G_{0} / G_{1}$ and/or $G_{2} / M$ through the upregulation of the cyclin-dependent kinase inhibitors p21 and p27, and the downregulation of Cdc2 and cyclin B1 [6]. Curcumin blocks signaling of growth factors, including human epidermal growth factor receptor-2 [7], plateletderived growth factor [8] and fibroblast growth factor [9]. It also blocks Wnt signalling [10]. Curcumin also has anti-invasive, antimetastastic, and antiangiogenic properties $[11,12]$. Therefore, curcumin is labeled as a multi-targeted drug. However, the systemic availability of curcumin remains negligible [13,14]. Hydrophobicity, low absorption, and rapid metabolism have been considered as reasons for curcumin's low bioavailability [2]. To address this issue, we synthesized a series of curcumin analogs and screened them. An analog named GO-Y030 was synthesized that possessed 30- to 50-fold enhanced antiproliferative potential against various types of cancers in vitro [15]. The molecular mechanisms of GOY030 closely resembled those of curcumin. Oral administration of GO-Y030 prevented the adenoma formation in the familial adenomatous polyposis (FAP) mouse

\section{Biomed Central}

(c) 2011 Kudo et al; licensee BioMed Central Ltd. This is an Open Access article distributed under the terms of the Creative Commons Attribution License (http://creativecommons.org/licenses/by/2.0), which permits unrestricted use, distribution, and reproduction in any medium, provided the original work is properly cited. 
model without any apparent toxicity [16]. It was also demonstrated that the number and the size of $\beta$-catenin-positive adenomas were reduced. In this case, bioavailability of GO YO30 was not an issue because it can be directly delivered to the gastrointestinal tract. However, GO-Y030 was hydrophobic and ineffective to the treatment of peritoneal carcinomatosis (PC) of gastric carcinoma in a mouse model. These observations led us to additionally synthesize hyperactive curcumin analogs in order to optimize their pharmacological potential.

\section{Results}

\section{Screening of the hyperactive growth inhibitors among} the newly synthesized curcumin analogs

Eighty eight curcumin analogs, including 69 species of DOPs, curcumin and 1,7-diaryl-1,6-heptadiene-3,5dione, and 17 species of non-DOP analogs were synthesized (Figure 1). The growth-suppressive potential of these compounds was then examined on cell panels composed of 16 types of cancers. These included gastric cancers (GCIY and SH10TC), colon cancers (HCT116, DLD1 and SW680), lung cancer (A549), pancreatic cancer (PK1), kidney cancer (ACHN), liver cancer (HUH7), ovarian cancer (OVK18), breast cancer (MCF7), skin cancer (A431), bile duct cancer (HuCCT1), thyroid cancer $(8505 \mathrm{c})$, melanoma (G361), and prostate cancer (PC3). The IC50 value of each analog was calculated against the individual cell line. These values were

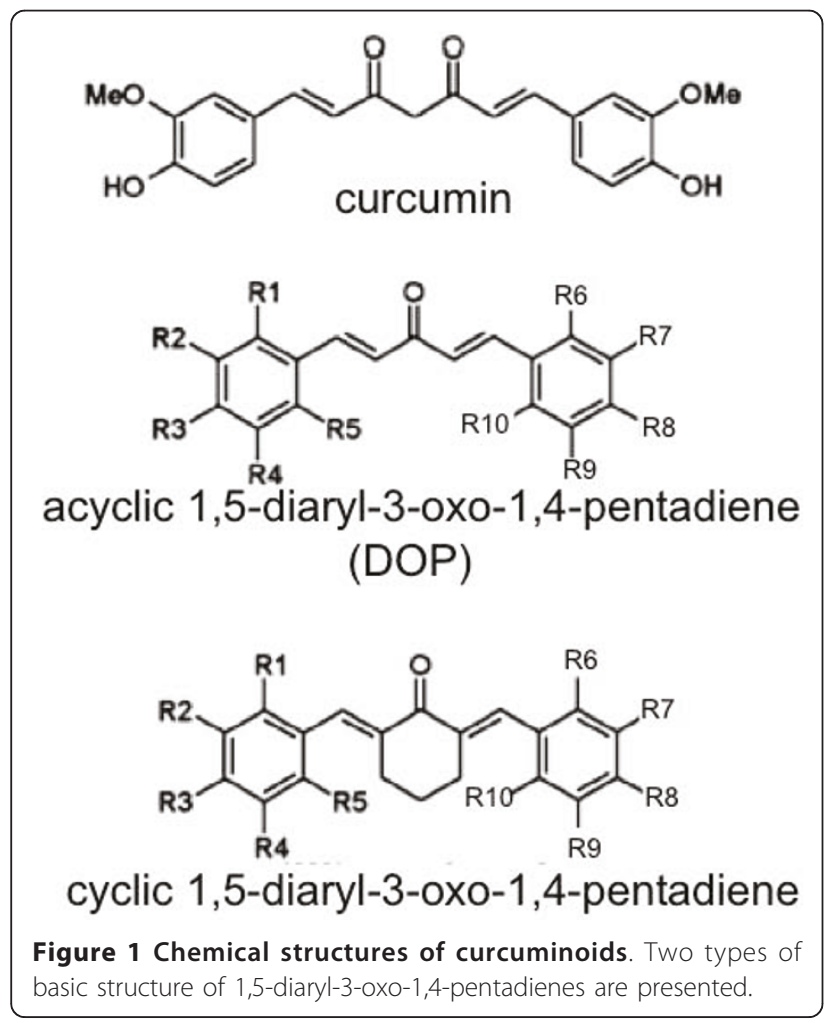

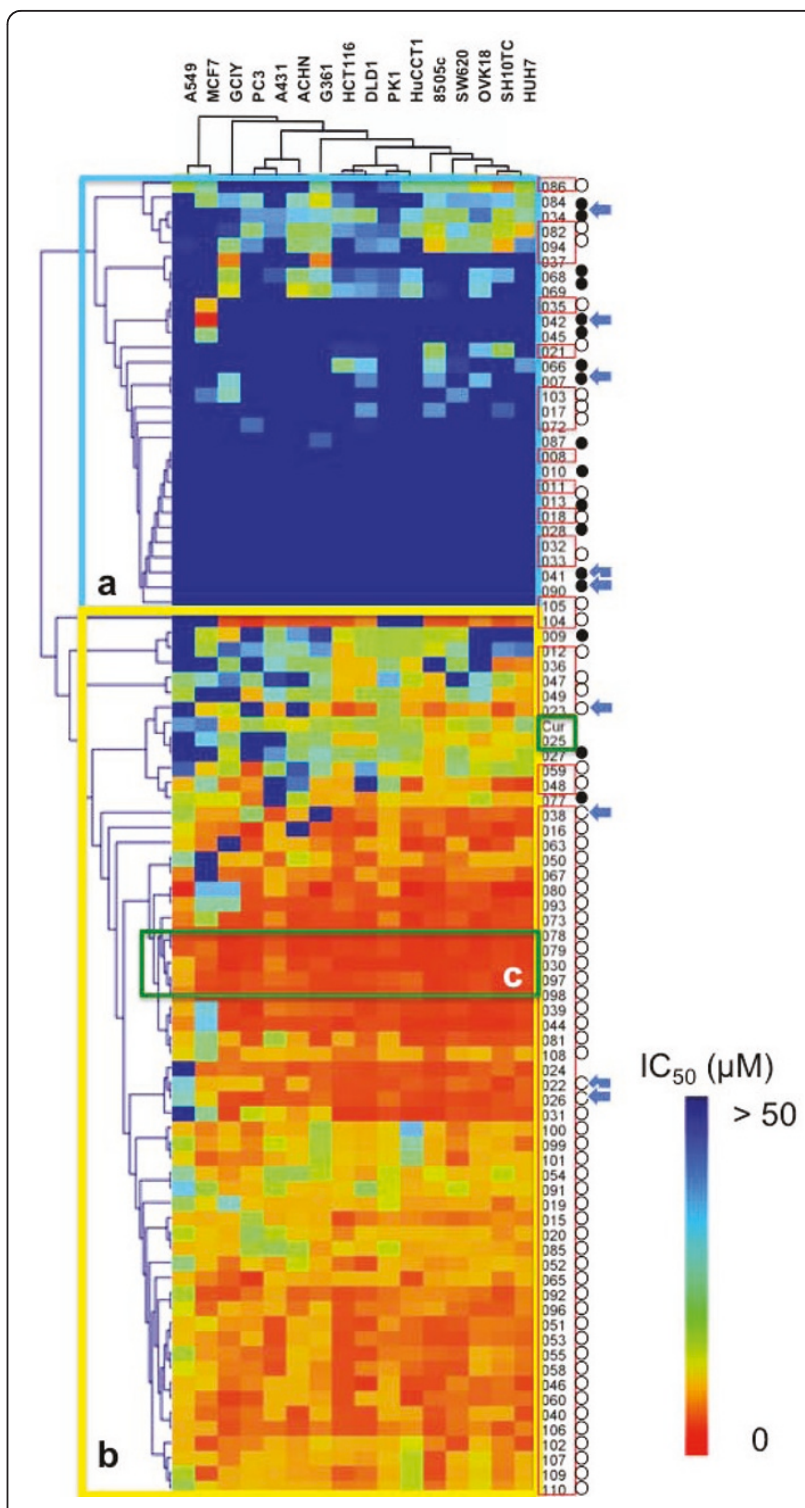

Figure 2 Cluster analysis of growth inhibition of 88 curcuminoids. The IC50 values are graded by coloring from red (0 $\mu \mathrm{M})$ to cyan $(50 \mu \mathrm{M})$. Eighty eight analogs are lined in

perpendicular, and 16 cancer cell lines are lined in horizontal. Group $\mathrm{a}$ is composed of the analogs with the high $\mathrm{IC}_{50}$ values, and Group b composed of analogs with the low $I C_{50}$ values, and Group c includes the analogs with the lowest $I C_{50}$ values. The analogs enclosed with red rectangle are DOPs, and the analogs attached with closed circle are non-DOPs. Acyclic DOPs are indicated by open circle. Blue arrows indicate the analogs of which predictive values of solubility are better than curcumin

analyzed using cluster analysis (Figure 2). Resulting from the pattern of inhibition, 88 curcuminoids were divided into 2 clusters; one is the high $\mathrm{IC}_{50}$ group (Group a) composed of 29 analogs, and the other is the low $\mathrm{IC}_{50}$ group (Group b) composed of 59 analogs. In detail of Group a, 51.7\% (15/29) of those are DOPs, 
whereas $91.5 \%$ (54/59) are DOPs in Group b. From the view point of DOPs, 55 out of $69(79.7 \%)$ belong to Group b. In this study, 64 species out of 69 analogs are classified to acyclic DOPs, and $81.3 \%$ species $(52 / 64)$ of acyclic DOPs belong to Group b. On the other hand, among 17 non-DOPs, $82.4 \%$ species belong to Group a. The analogs that exhibited the strongest potential against all of the cell lines were clustered in Group c (Figure 2). This cluster included GO-Y078, 079, 030, 097, and 098. All of these compounds had at least 10 times higher growth-suppressive potential than curcumin. For example, the value of GO-Y030 reached 76 times, GO-Y078 84 times, and GO-Y079 29 times higher than curcumin against GCIY. In the solution, as each ethoxyethyl group of GO-Y079 and 097 is predicted to be hydrolyzed in an acidic environment, and GO-Y079 and GO-Y097 can convert themselves to 078 and 098, respectively. Essentially, GO-Y078 is nearly identical to 079 , and 098 to 097 , just as shown in the cluster analysis. For this reason, we excluded GO-Y079 and 097 from further examination. In this analysis, it has been shown A549 and MCF7 were rather resistant to curcumin analogs among them (Figure 2).

\section{Improvement in solubility of curcumin analogs}

The ADME profiles of 100 curcuminoids, including virtual analogous compounds were determined in silico. Concerning solubility of analogs, the predicted values of top 15 are represented in the bar graph (Figure 3a). Analogs, that bear the improved solubility than curcumin and belong to Group b at the same time, are 8 compounds including GO-Y078 and Y098. The predictive values of them are 1.98 and 2.93 times higher than curcumin, respectively. The highest predictive value is $11.7 \mathrm{mg} / \mathrm{L}$ of GO-Y038,

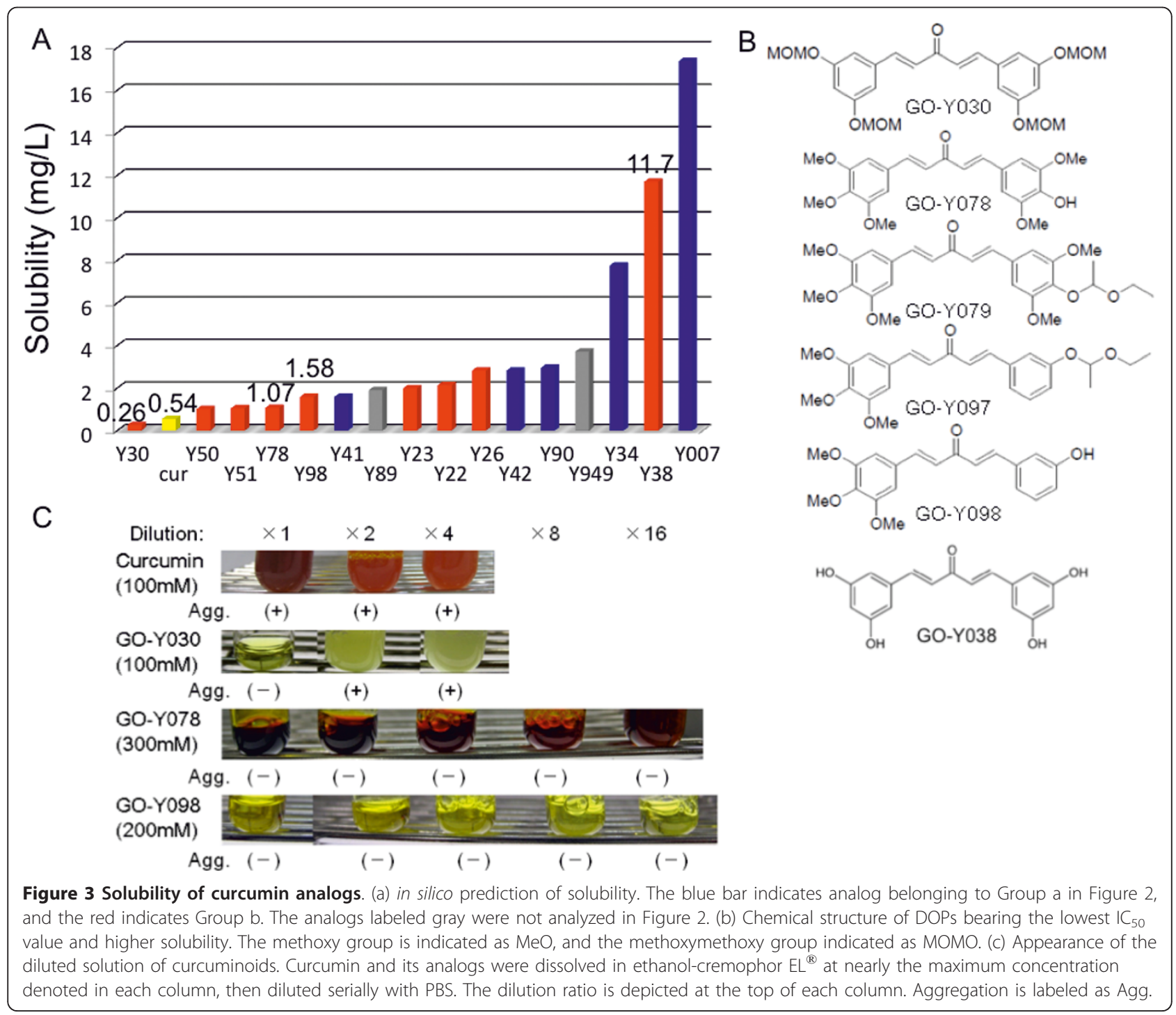


which is 21.7 times higher than curcumin. As for GOY030, the solubility is 0.48 times lower than curcumin. Then, we practically examined the solubility of curcumin, GO-Y030, 078, and 098 (Figure 3b). These compounds were first dissolved in ethanol-cremophor $\mathrm{EL}^{\circledR}$ and then diluted in PBS. Concentration of GO-Y078 in ethanol-cremophor $\mathrm{EL}^{\circledR}$ reached the highest level among them at 300 $\mathrm{mM}$. While there were no aggregations seen in GO-Y078 and 098 solutions after 4 serial dilutions, the appearance of aggregation was observed in curcumin and GO-Y030 solutions immediately after the first dilution (Figure 3c). In fact, GO-Y078 and 098 showed increased solubility, as predicted.

Growth suppression and apoptosis induction potencies of GO-Y030, 078, and 098

To examine the growth suppression of 3 DOP analogs, GO-Y030, Y078, and Y098 clustered in Group c, the analysis of cell cycle progression in HCT116 cells was conducted by flow cytometry. Analysis of cell cycle progression indicated the increase of the $\mathrm{subG}_{1}$ fraction. Those treated with GO-Y030, 078, and 098 at $2 \mu \mathrm{M}$ were $27.2 \%, 17.1 \%$, and $19.8 \%$, respectively. These values were 6.0, 3.8, and 4.4 times higher than curcumin at the same concentration (Figure 4a). GO-Y030, 078, and 098 may have a stronger potency to induce apoptosis than curcumin. To further elucidate this observation, caspase cleavage reactions were assessed. At a dose of $2 \mu \mathrm{M}$, the amount of cleaved product in GO-Y030, 078, and 098 treatments were $2.12 \pm 0.07,3.47 \pm 0.84$ and $1.67 \pm 0.19$ times higher, respectively, than curcumin. GO-Y030, 078, and 098 were also considered to be potent inducers of apoptosis (Figure 4b).

\section{GO-Y030, 078, and 098 can induce apoptosis through} numerous mechanisms just as curcumin

Generally, cancer cells have some breakdown in key apoptosis induction mechanisms. These include the

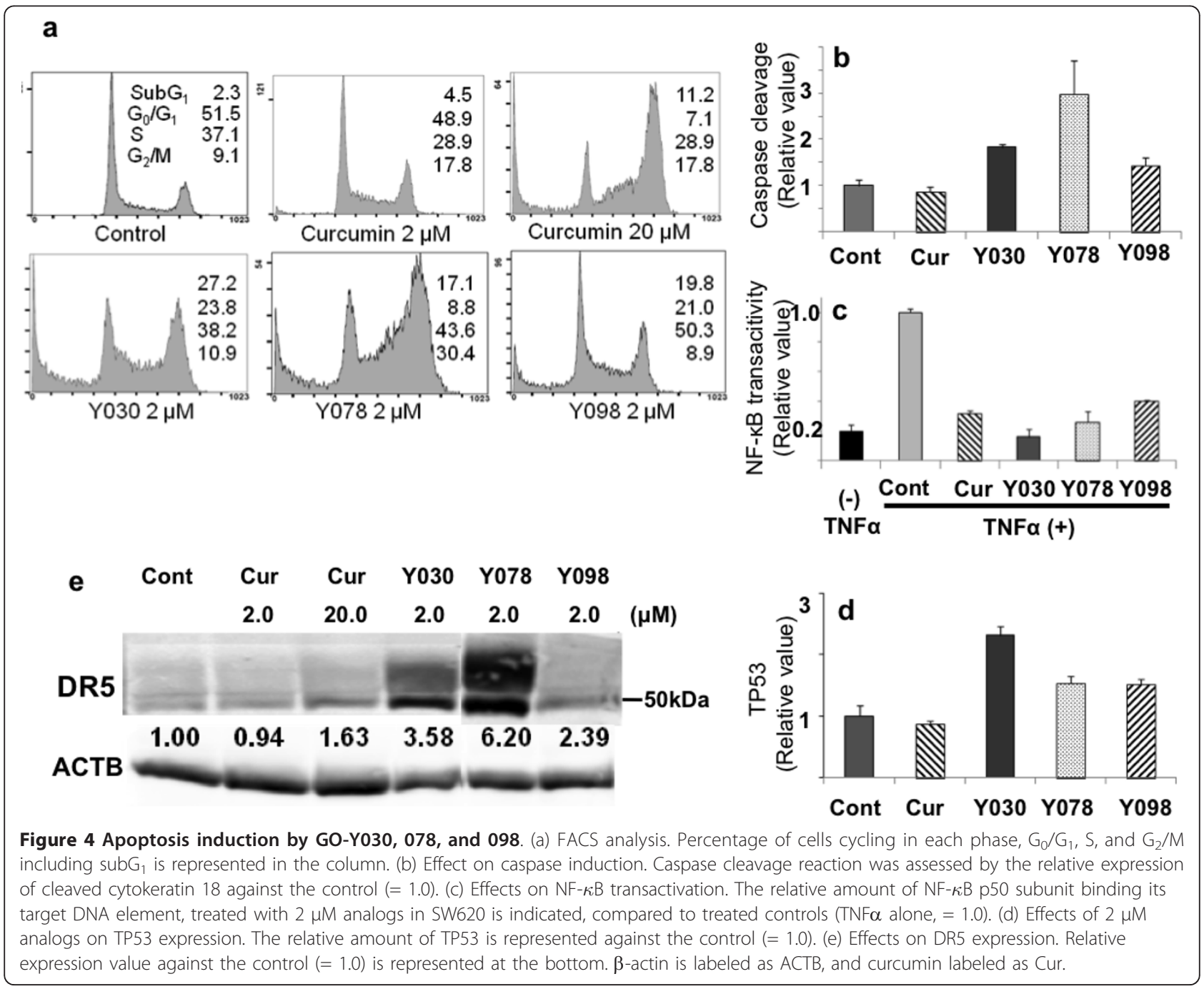


inactivation of a cohort of NF- $\kappa \mathrm{B}$ target genes, as well as inactivation of the TP53 pathway, and extrinsic apoptotic pathways. Hyperactivated NF- $\kappa \mathrm{B}$ in cancer cells leads to protection from apoptotic cell death via its targets including IAPs. It is well documented that curcumin can inhibit NF- $\kappa \mathrm{B}$ transactivation $[3,4]$. The inhibitory effects of NF- $\kappa \mathrm{B}$ transactivation in $3 \mathrm{DOP}$ analogs, were examined by ELISA. In this assay, NF- $\kappa \mathrm{B}$ transactivation was estimated by the amount of p50 subunit binding to its DNA consensus sequence. NF- $\kappa \mathrm{B}$ p50 activates a set of target genes in response to certain inflammatory signals, such as TNF $\alpha$. In this study, curcumin and DOP analogs decreased TNF $\alpha$-induced NF$\kappa \mathrm{B}$ transactivation at a dose of $2 \mu \mathrm{M}$. The relative values of transactivation with curcumin, GO-Y030, 078, and 098 were $0.32 \pm 0.02,0.16 \pm 0.05,0.26 \pm 0.07$, and 0.40 \pm 0.01 compared to the control $(=1.0$ ) (Figure $4 \mathrm{c}$ ). These DOPs were shown to have comparative potency to curcumin in the inhibition of NF- $\kappa \mathrm{B}$ transactivation.

The p53 gene is altered in nearly half of human cancers, usually by point mutations, and results in the loss of ability to induce pro-apoptotic genes. Following $24 \mathrm{~h}$ of treatment, TP53 induction was assessed in HCT116 cells bearing wild-type p53 as a control. After treatment with a $2 \mu \mathrm{M}$ dose, the TP53 expression in curcumintreated cells was similar to the control level, while the levels of TP53 in GO-Y030, 078, and 098 were increased $2.32,1.54$, and 1.52 times higher, respectively, than the control. (Figure 4d). Activating certain death receptors (DR), particularly DR4 and DR5, has been shown to selectively kill cancer cells while sparing normal cells [21]. Curcumin activates DR5, but does not activate DR4 [22]. DR5 is regulated by either a p53-dependent or -independent mechanism [23]. DR5 overexpression induces ligand-independent apoptosis [24]. The expression of DR5 was estimated in DLD-1 bearing mutant TP53, by Western blotting. After $24 \mathrm{~h}$ of incubation, the expression of DR5 was increased to $3.58,6.20$, and 2.39 times higher than the control, in GO-030, 078, and 098 treated cells, respectively (Figure 4e). It was shown that the apoptosis induction potency, including overexpression of TP53 and DR5 was improved, but NF- $\kappa$ B inhibition was not.

\section{Therapeutic effects of DOP analogs in an experimental mouse model}

According to the data concerning solubility described above, GO-Y078 and 098 may have improved bioavailability. Initially, the toxicity of these compounds was examined in vitro. We examined the growth suppression of the primary normal liver cells, hNHeps ${ }^{\circledR}$ with GOY030, 078, and 098. They do not affect survival nor have any toxic effects to hNHeps ${ }^{\circledR}$ at doses as high as $50 \mu \mathrm{M}$. Then, we conducted a single IP injection, and determining the maximum tolerable dose (MTD). The MTDs of GO-Y030 and 078 were determined to be 474.5 and $400 \mathrm{mg} / \mathrm{kg}$, respectively. Administration of these doses did not affect the animals for over 1 month. However, the MTD of GO-Y098 was rather toxic at a dose below $204 \mathrm{mg} / \mathrm{kg}$, and this compound was excluded from further examination. The compounds were then applied to the mouse model of PC with GCIY, a gastric cancer notorious for its rapid progression [25]. The PC progresses very rapidly, and increases in ascites fluid and comparative weight gain continues until the animal dies. As a result, mortality occurs within 1 month following tumor inoculation. Experimental treatment was carried out as shown in Figure 5a. The IP administration of curcumin had no effect on the PC model, where the average body weight of untreated group reached to $164.0 \pm 20.8 \%$ of that before treatment, whereas that treated with curcumin to $150.2 \pm$ 24.0\% ( $\mathrm{P}=0.18)$. GO-Y030 had a significant antitumor effect on PC progression ( $\mathrm{p}<0.001$, Figure $5 \mathrm{~b}$ ), but no survival benefit was observed (Figure 5c). Similar to that of GO-Y030, GO-Y078 showed a significant suppression of $\mathrm{PC}$ progression. At the 17 th day after the first treatment, the average body weight of the untreated group was $35.9 \pm 2.6 \mathrm{~g}$ due to increased ascites fluid accumulation, while that of the treated group was significantly less at $29.1 \pm 2.5 \mathrm{~g}(\mathrm{p}=0.003$, Figure $5 \mathrm{~d})$. The average body weight before inoculation was $28.4 \pm 0.9 \mathrm{~g}$, therefore the ascites fluid accumulation was considered to be completely suppressed by GO-Y078 treatment. Survival time of the untreated group $(n=4)$ ranged from 21 to 23 days, with a median survival time (MST) of 22 days. Survival time of the GO-Y078 treated group $(n=6)$ ranged from 25 to 109 days, with a MST of 30.5 days. Treatment with GO-Y078 led to an approximate $40 \%$ increase in survival time. Log-rank analysis significantly indicated a survival benefit of GO-Y078 treatment ( $\mathrm{p}=$ 0.01 , Figure $5 \mathrm{e})$. Judging from these results, the bioavailability of GO-Y078 is significantly higher than GOY030. In order to examine why the survival was limited in the GO-Y078 treated mice, despite the fact that the ascites fluid accumulation disappeared completely. When necropsies were performed, in nearly all of the GO-Y078-treated mice, residual cancer cells were observed to colonize around the stomach, pancreas, bile duct, and duodenum. Direct invasion to the pancreas, liver, and duodenum was thought to be the causes of death.

\section{Discussion}

DOP analogs possess common structural feature, that is an electrophilic $\alpha, \beta$-unsaturated ketone, which reacts with nucleophilic groups through the Michael addition. Then, the ketone binds covalently with thiol groups of 


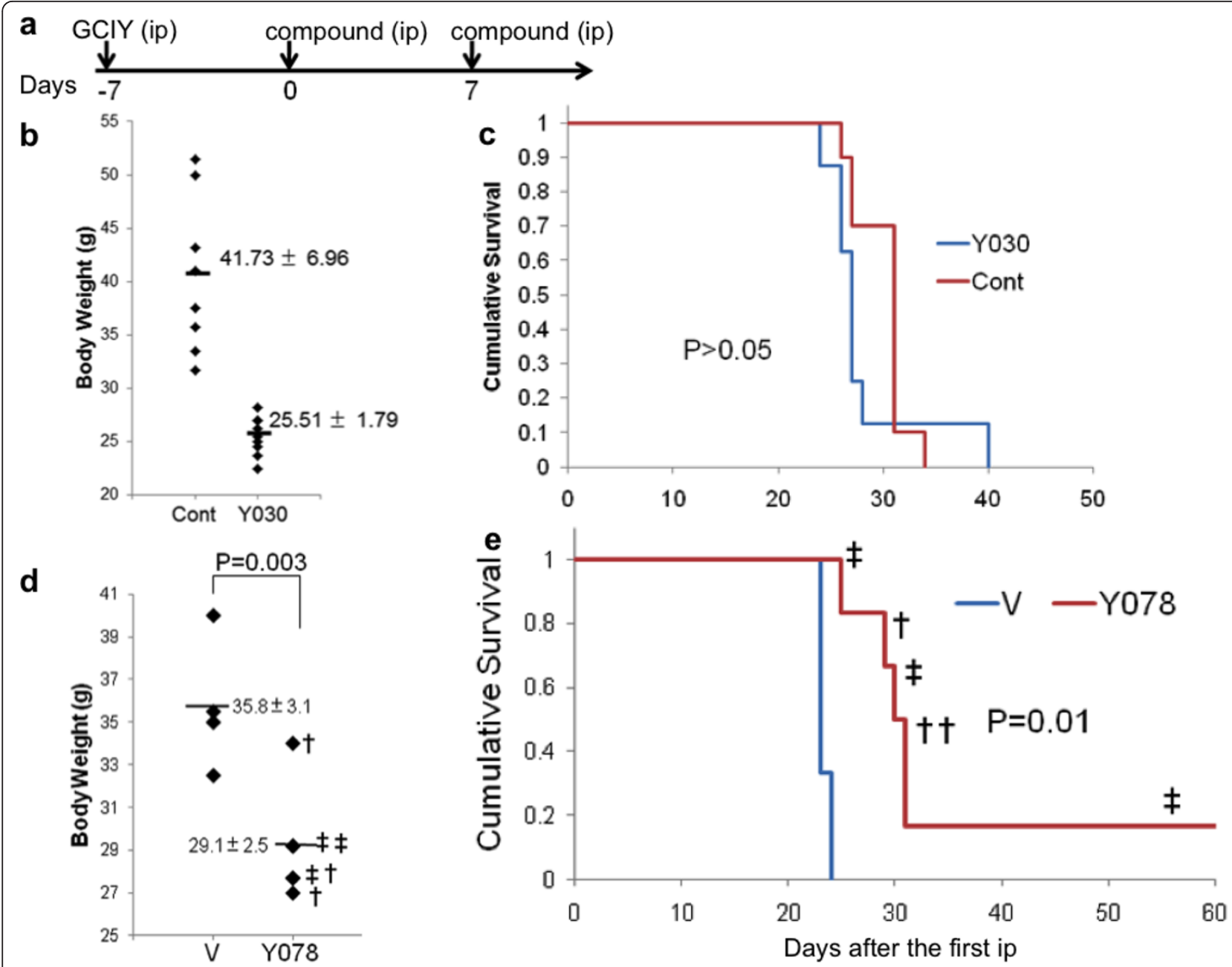

Figure 5 Experimental treatment with GO-Y078 and $\mathbf{0 3 0}$ in mice. (a) The schedule of experimental treatment. (b) Antitumor effect of GOY030. The body weight corresponding to the progression of PC, were evaluated. V, ethanol-cremophor, 30; Y030 $155 \mathrm{mg} / \mathrm{kg}$. (c) Survival benefit of GO-Y030. (d) Antitumor effect of GO-Y078. The body weight corresponding to the progression of PC was evaluated at the 17th day. V, ethanol-cremophor; †, $133 \mathrm{mg} / \mathrm{kg} ;$ †, $266 \mathrm{mg} / \mathrm{kg}$ (e) Survival benefit of GO-Y078.

cysteine residues in different proteins [26]. As thiol group does not exist in DNA, curcuminoids do not attach DNA itself, different from the cytotoxic chemotherapeutic agents. Two independent proteins can be alkylated through two $\alpha, \beta$-unsaturated double bonds in DOP that stretch from the center ketone moiety toward both ends [27]. In this alkylation, an association of a cysteine protease, such as caspase, and its target protein may assist in the catalytic reaction of caspase, resulting in target degradation. In our previous study, we observed that DOP analogs increased caspase activity, and a caspase inhibitor inhibited the degradation of $\beta$ catenin or KRAS by DOP analogs [15]. Although this contention remains unproven, perhaps this center structure of DOP confering Michael addition is responsible for the multi-targeted nature of curcuminoids. Contribution to enhanced potency by the alteration from a diketone to a monoketone also remains to be elucidated. Shortening the chemical distance by alkylation through conversion from a di- to monoketone structure may result in an enhanced potential. Acyclic DOP analogs have an enhanced cytotoxicity [27]. Substitution in each aromatic ring could improve solubility and the resultant pharmaceutical potency. As the symmetry of the attachment group of each aromatic ring is not necessary, further improvement of solubility may be achievable by various types of substitution in this portion [17]. The hydrophilic nature and total positioning of the attached groups in a compound can reflect the potency of the analogs. In this study, the maximum $\mathrm{IC}_{50}$ value of $\mathrm{DOP}$ analogs reached a level that was 84 times lower than curcumin. The data, that curcuminoids could only affect the malignant growth, lead to the hypothesis that the growth-suppressive potential of these analogs depends 
on the degradation of oncoproteins that are overexpressed in malignant cells. That might be a reason why curcuminoids are rather safe to normal cells.

Furthermore, it has been shown that some of DOP analogs induce more cell death to cancer cells, compared with curcumin. Apoptosis related proteins, such as TP53 and DR5 were up-regulated with DOP analogs more efficiently than curcumin. However, the precise mechanisms of up-regulation are still unknown [28]. Cancer cells invent numerous ways to inactivate many of the apoptotic pathways. The pluripotent apoptosis induction by curcuminoids could overcome complexity of antiapoptosis in cancer cells. One of DOP analog, GO-Y078 has shown survival benefit in mouse PC model for the first time. That could not be achieved by GO-Y030. Although these two DOPs presented the same level of $\mathrm{IC}_{50}$ values, the predicted solubility of GO-Y078 was $1.98 \mathrm{mg} / \mathrm{L}$, whereas that of GO-Y030 was $0.26 \mathrm{mg} / \mathrm{L}$. In fact, GO-Y030 was less soluble compared with GO-Y078. The difference of solubility between GO-Y030 and GO-Y078 probably influences the difference in their survival benefits in the PC models. Moreover, additional times of administration of GO-Y078 may lead to further improved survival. Combined treatment with conventional cytotoxic agents, such as 5 -FU and taxanes, or a DR5 ligand, for example, tumor necrosis factor-related apoptosis-inducing ligand (TRAIL), may synergistically induce better outcomes [22]. Recently, off-target toxicity has become a problem in molecular-targeted medicines which have already been approved $[29,30]$. Some of the potent DOP analogs may not be as safe as dietary curcumin, shown with GOY098, and we should be cautious against that during development.

\section{Conclusions}

Synthesis of DOP analogs represented by GO-Y078 can overcome low bioavailability of curcumin, yielding good lead compounds for new cancer chemotherapeutic agents bearing multi-target properties.

\section{Methods}

\section{Chemicals}

Chemical synthesis, physical properties and molecular formulas of the newly synthesized curcumin analogs have been previously described $[15,17]$. See additional file 1: Physical properties of new curcumin analogs. Curcumin (Sigma-Aldrich) and its analogs were dissolved in DMSO at $10-50 \mathrm{mmol} / \mathrm{L}$ as a stock solution. Cremophor $\mathrm{EL}^{\circledR}$ was purchased from Sigma-Aldrich.

\section{Cell lines}

GCIY, HCT116, DLD1, SW680, A549, PK1, ACHN, HUH7, OVK18, MCF7, 8505c, G361, and PC3 were obtained from the Cell Resource Center for Biomedical Research (Institute of Development, Aging and Cancer, Tohoku University, Sendai, Japan). ACHN was obtained from the American Type Culture Collection (ATCC). HuCCT1, SH10TC, and A431 were obtained from Riken Cell Bank Normal human primary hepatocytes $\left(\right.$ hNHeps ${ }^{\circledR}$ ) were purchased from Lonza. Cells were cultured in RPMI 1640 medium containing 10\% fetal bovine serum.

\section{Growth-suppression analysis}

Growth-suppressive effects of the derivative compounds were measured in different cancer cell lines for $72 \mathrm{~h}$. Cell viability was assayed by quantifying the uptake and digestion of 2-(2-methoxy-4-nitrophenyl)-3-(4-nitrophenyl)-5-(2,4-disulfophenyl)-2H-tetrazolium monosodium salt according to the manufacturer's instructions (Dojindo Laboratories) by 96-well plate reader, SpectraMax M2e (Molecular Devices). The percentage cell growth of the control, which was treated with $1 \%$ DMSO alone, was calculated and plotted, and the mean growth-inhibitory concentration $\left(\mathrm{IC}_{50}\right)$ value was then determined.

\section{Cluster analysis}

Clustering analysis among analogs was performed with MultiExperiment Viewer software $(\mathrm{MeV})$ from the institute for Genomic Research at that time [18].

\section{Cell Cycle analysis}

Cell cycle phase was determined by fluorescence-activated cell sorting analysis. Cells from the HCT116 cell line were inoculated into 6-well plates at a concentration of $5 \times 10^{5}$ per well, exposed to the derivative compounds at various concentrations, cultured for $24 \mathrm{~h}$, and collected and sorted using a Cytomics FC500 Flow Cytometry System (Beckman Coulter, Inc.) as previously described [19]. The percentage of each cell fraction corresponding to the subG $G_{1}, G_{0} / G_{1}, S$, and $G_{2} / M$ phases was calculated using WinCycle Software (Beckman).

\section{Expression analysis}

HCT116 cells treated by the analogs were cultured for $24 \mathrm{~h}$, collected and lysed with lysis buffer $(500 \mathrm{mM}$ Tris- $\mathrm{HCl} \mathrm{pH}$ 7.5, $100 \mathrm{mM} \mathrm{NaCl}, 2 \mathrm{mM}$ EDTA, $1 \mathrm{mM}$ sodium orthovanadate, and 1\% NP-40). After centrifuging samples, the supernatant fraction was transferred to a fresh tube. Samples were mixed with $5 \times$ SDS sample buffer (312.5 mM Tris- $\mathrm{HCl} \mathrm{pH} 6.8,50 \%$ Glycerol, $25 \%$ 2-mercaptoethanol, $12.5 \%$ SDS, and $0.1 \%$ bromophenol blue) before western blotting. Antibodies used for Western blotting were anti-beta-actin monoclonal antibody (A2066, Sigma-Aldrich), anti-DR5 monoclonal antibody (Santa Cruz Biotechnology), and Alexa Fluor 
680 (Invitrogen). Membranes were scanned and semiquantification was conducted using the Odyssey detection system (LI-COR). Expression of TP53 was measured using P53 ELISA kit (Promokine) according to the manufacturer's instructions. Samples were collected using the same method as Western blotting. Twenty micrograms of protein were incubated in wells coated with anti-human p53 antibody. Biotin-conjugated antihuman p53 antibody was then added. After incubation, streptavidin conjugated with horse radish peroxidase (HRP) was added. Tetramethyl-benzine, which reacts with HRP, was then added and the absorbance was measured at $450 \mathrm{~nm}$ by SpectraMax M2e.

\section{Caspase cleavage reaction}

HCT116 cells were inoculated at a concentration of $1 \times$ $10^{4}$ per well in a 96-well plate, cultured until cells were semi-confluent, and treated with the analogs for $24 \mathrm{~h}$. Serum from each sample was collected, duplicated, and assessed by ELISA using HRP-conjugated M30 monoclonal antibody (M30-Apoptsense ${ }^{\mathbb{B}}$ ELISA, Peviva) according to the manufacturer's instructions. Anti-M30 monoclonal antibody recognizes a soluble cytokeratin18Asp396 molecule that becomes exposed after cleavage by caspases (caspase3, 6, 7, and 9) during apoptosis. The absorbance was measured by SpectraMax M2e at 450 $\mathrm{nm}$. These analyses were conducted in triplicate.

\section{NF- $\kappa$ B transactivation}

$\mathrm{NF}-\kappa \mathrm{B}$ transactivation was measured by ELISA using a NF $\kappa \mathrm{B}$ p50 Transcription Factor Assay kit (Thermo Scientific) according to the manufacturer's instructions. SW620 cells were treated with the compounds with or without Tumor Necrosis Factor $\alpha$ (TNF $\alpha)(10 \mathrm{ng} / \mathrm{ml}$, Sigma) for 6 hours, washed with phosphate buffered saline (PBS), and lysed with provided M-PER ${ }^{\circledR}$ lysis buffer (Thermo Scientific). Whole-cell extracts containing 20 $\mu \mathrm{g}$ of protein were added with biotinylated-NF- $\kappa \mathrm{B}$ consensus DNA sequence to a streptavidin-coated 96-well plate. Sample wells were incubated with the monoclonal antibody against NF- $\kappa \mathrm{B}$ p50, and then incubated with HRP-conjugated secondary antibody. Luminol/enhancer solution and stable peroxide solution were added and $\mathrm{NF}-\kappa \mathrm{B}$ transactivation was detected using an LMax Luminometer (Molecular Devices).

\section{In silico ADME analysis}

Absorption, distribution, metabolism, excretion (ADME), and solubility, were analyzed in silico by ADMEWORKS/ Predictor (Fujitsu Kyushu Systems Ltd.). This analysis is commercially available and depends on a quantitative structure-activity relationship [20]. Solubility was calculated by the linear multiple regression equation and indicated by the predicted value.

\section{Animal experiment}

C57BL/6J mice were obtained from CLEA Japan, Inc. and KSN Slc (nu/nu) mice were obtained from Japan SLC. The injection solution was prepared by first dissolving the analogs with ethanol-cremophor $\mathrm{EL}^{\circledR}(1: 1)$ and then diluting the solution 1:8 with PBS. C57BL/6J mice were used for the assessment of toxicity of the C5-curcuminoids. The maximum tolerable dose (MTD) was determined by a single intraperitoneal (IP) administration. Therapeutic experiments were conducted with the IP administration of $155 \mathrm{mg} / \mathrm{kg}$ of GO-Y030 (total volume $1.0 \mathrm{~mL}$ ). IP administration of GO-Y078 was conducted with $133 \mathrm{mg} / \mathrm{kg}$ or $266 \mathrm{mg} / \mathrm{kg}$ doses. Ethanol-cremophor EL ${ }^{\circledR}$ alone diluted 1:8 with PBS was injected as a control. The PC model was established by inoculating $5 \times 10^{6}$ GCIY cells into the abdominal cavities 6-week-old male $\mathrm{KSN} / \mathrm{Slc}$ mice, 1 week prior to treatment with the 1,5-diaryl-3-oxo-1,4-pentadiene (DOP) analogs. Pathological examination including hematoxylin-eosin staining was performed as previously described [16]. All animal experiments were performed in accordance with approved institutional animal use guidelines (Tohoku University) based on international guideline.

\section{Statistic analysis}

Each experiment was conducted three times, unless specified otherwise. Individual values were compared by Student's $t$-test. The survival curve was drawn using the Kaplan-Meier method and compared using the log rank test.

\section{Additional material}

Additional file 1: Physical properties of new curcumin analogs. The physical properties and molecular formula of the analogs are described in the file.

\section{List of abbreviations}

NF-kB: nuclear factor-kB; FAP: familial adenomatous polyposis; PC: peritoneal carcinomatosis; HRP: horse radish peroxidase; TNFa: Tumor Necrosis Factor a ADME: Absorption, distribution, metabolism, excretion; DOP: 1,5-diaryl-3-oxo1,4-pentadiene; DR: death receptors; MTD: maximum tolerable dose

\section{Acknowledgements}

This work was supported by a Grant-in-Aid for Scientific Research (Category C, 22501041) from the Japan Society for the Promotion of Science (to HO,

$\mathrm{YI}$, and HS) and Collaborative Research Fund (Kanazawa University and Akita University) (to $\mathrm{Yl}$, and $\mathrm{HS}$ ).

\section{Author details}

'Dept. Clinical Oncology, Institute of Development, Aging, and Cancer, Tohoku University, Seiryo-cho 4-1, Aoba-ku, Sendai, Japan. '2Dept. Clinical Oncology, University Hospital, Tohoku University, Seiryo-cho 1-1, Aoba-ku, Sendai, Japan. ${ }^{3}$ Dept. Organic Chemistry, Graduate School of Pharmaceutical Science, Tohoku University, Aoba 6-3, Aramaki, Aoba-ku, Sendai, Japan.

${ }^{4}$ Division of Clinical Pathology, Faculty of Medicine, Akita University, Hondo 1- 
1-1, Akita, Japan. ${ }^{5}$ Dept. Clinical Oncology, Faculty of Medicine, Akita University, Hondo1-1-1, Akita, Japan.

\section{Authors' contributions}

CK conducted all experiments and statistical analysis. AS and $\mathrm{HO}$ participated in cell culture and animal experiments. HY and YI designed and synthesized all of the analogs assayed in this study. Cl participated in design of the study. HS designed this study and described the manuscript. All authors read the final version of the manuscript and approved.

\section{Competing interests}

The authors declare that they have no competing interests.

Received: 14 March 2011 Accepted: 28 May 2011

Published: 28 May 2011

\section{References}

1. Tan G, Gyllenhaal C, Soejarto DD: Biodiversity as a source of anticancer drugs. Curr Drug Targets 2006, 7:265-277.

2. Goel A, Kunnumakkara AB, Aggarwal BB: Curcumin as "Curecumin": from kitchen to clinic. Biochem Pharmacol 2008, 75:787-809.

3. Singh S, Aggarwal BB: Activation of transcription factor NF-kappa B is suppressed by curcumin (diferuloylmethane). J Biol Chem 1995, 270:24995-25000.

4. Surh YJ, Han SS, Keum YS, Seo HJ, Lee SS: Inhibitory effects of curcumin and capsaicin on phorbol ester-induced activation of eukaryotic transcription factors, NF-kappaB and AP-1. Biofactors 2000, 12:107-112.

5. Jaiswal AS, Marlow BP, Gupta N, Narayan S: Beta-catenin-mediated transactivation and cell-cell adhesion pathways are important in curcumin (diferuylmethane)-induced growth arrest and apoptosis in colon cancer cells. Oncogene 2002, 21:8414-8427.

6. Park MJ, Kim EH, Park IC, Lee HC, Woo SH, Lee JY, Hong YJ, Rhee CH, Choi SH, Shim BS, Lee SH, Hong Sl: Curcumin inhibits cell cycle progression of immortalized human umbilical vein endothelial (ECV304) cells by up-regulating cyclin-dependent kinase inhibitor, p21WAF1/CIP1, p27KIP1 and p53. Int J Oncol 2002, 21:379-383.

7. Hong RL, Spohn WH, Hung MC: Curcumin inhibits tyrosine kinase activity of p185neu and also depletes p185neu. Clin Cancer Res 1999, 5:1884-1891.

8. Yang X, Thomas DP, Zhang X, Culver BW, Alexander BM, Murdoch WJ, Rao MN, Tulis DA, Ren J, Sreejayan N: Curcumin inhibits platelet-derived growth factor-stimulated vascular smooth muscle cell function and injury-induced neointima formation. Arterioscler Thromb Vasc Biol 2006, 26:85-90.

9. Mohan R, Sivak J, Ashton P, Russo LA, Pham BO, Kasahara N, Raizman MB, Fini ME: Curcuminoids inhibit the angiogenic response stimulated by fibroblast growth factor-2, including expression of matrix metalloproteinase gelatinase B. J Biol Chem 2000, 275:10405-10412.

10. Ryu MJ, Cho M, Song JY, Yun YS, Choi IW, Kim DE, Park BS, Oh S: Natural derivatives of curcumin attenuate the $\mathrm{Wnt} /$ beta-catenin pathway through down-regulation of the transcriptional coactivator p300. Biochem Biophys Res Commun 2008, 377:1304-1308.

11. Hahm ER, Gho YS, Park S, Park C, Kim KW, Yang CH: Synthetic curcumin analogs inhibit activator protein-1 transcription and tumor-induced angiogenesis. Biochem Biophys Res Commun 2004, 321:337-344.

12. Menon LG, Kuttan R, Kuttan G: Anti-metastatic activity of curcumin and catechin. Cancer Lett 1999, 141:159-165.

13. Garcea G, Berry DP, Jones DJ, Singh R, Dennison AR, Farmer PB, Sharma RA, Steward WP, Gescher AJ: Consumption of the putative chemopreventive agent curcumin by cancer patients: assessment of curcumin levels in the colorectum and their pharmacodynamic consequences. Cancer Epidemiol Biomarkers Prev 2005, 14:120-125.

14. Sharma RA, Euden SA, Platton SL, Cooke DN, Shafayat A, Hewitt HR, Marczylo TH, Morgan B, Hemingway D, Plummer SM, Pirmohamed M, Gescher AJ Steward WP: Phase I clinical trial of oral curcumin: biomarkers of systemic activity and compliance. Clin Cancer Res 2004, 10:6847-6854

15. Ohori H, Yamakoshi H, Tomizawa M, Shibuya M, Kakudo Y, Takahashi A, Takahashi S, Kato S, Suzuki T, Ishioka C, Iwabuchi Y, Shibata H: Synthesis and biological analysis of new curcumin analogues bearing an enhanced potential for the medicinal treatment of cancer. Mol Cancer Ther 2006, 5:2563-2571.
16. Shibata H, Yamakoshi H, Sato A, Ohori H, Kakudo Y, Kudo C, Takahashi Y, Watanabe M, Takano H, Ishioka C, Noda T, Iwabuchi Y: Newly synthesized curcumin analog has improved potential to prevent colorectal carcinogenesis in vivo. Cancer Sci 2009, 100:956-960.

17. Yamakoshi $\mathrm{H}$, Ohori $\mathrm{H}$, Kudo $\mathrm{C}$, Sato A, Kanoh N, Ishioka C, Shibata H, Iwabuchi Y: Structure-activity relationship of C5-curcuminoids and synthesis of their molecular probes thereof. Bioorg Med Chem 2010, 18:1083-1092.

18. The Institute for Genomic Research (TIGR):[http://www.tigr.org], TIGR was absorbed under the J. Craig Venter Institute name in 2007.

19. Kakudo Y, Shibata H, Otsuka K, Kato S, Ishioka C: Lack of correlation between p53-dependent transcriptional activity and the ability to induce apoptosis among 179 mutant p53s. Cancer Res 2005, 65:2108-2114.

20. Hayashi M, Kamata E, Hirose A, Takahashi M, Morita T, Ema M: In silico assessment of chemical mutagenesis in comparison with results of Salmonella microsome assay on 909 chemicals. Mutat Res 2005, 588:129-135.

21. Elrod HA, Sun SY: Modulation of death receptors by cancer therapeutic agents. Cancer Biol Ther 2008, 7:163-173.

22. Jung EM, Lim JH, Lee TJ, Park JW, Choi KS, Kwon TK: Curcumin sensitizes tumor necrosis factor-related apoptosis-inducing ligand (TRAIL)-induced apoptosis through reactive oxygen species-mediated upregulation of death receptor 5 (DR5). Carcinogenesis 2005, 26:1905-1913.

23. Lim JH, Park JW, Choi KS, Park YB, Kwon TK: Rottlerin induces apoptosis via death receptor 5 (DR5) upregulation through CHOP-dependent and PKC delta-independent mechanism in human malignant tumor cells. Carcinogenesis 2009, 30:729-736.

24. Sheridan JP, Marsters SA, Pitti RM, Gurney A, Skubatch M, Baldwin D, Ramakrishnan L, Gray CL, Baker K, Wood WI, Goddard AD, Godowski P, Ashkenazi A: Control of TRAIL-induced apoptosis by a family of signaling and decoy receptors. Science 1997, 277:818-821.

25. Nakanishi H, Mochizuki Y, Kodera Y, Ito S, Yamamura Y, Ito K, Akiyama S, Nakao A, Tatematsu M: Chemosensitivity of peritoneal micrometastases as evaluated using a green fluorescence protein (GFP)-tagged human gastric cancer cell line. Cancer Sci 2003, 94:112-118.

26. Lopez-Lazaro M: Anticancer and carcinogenic properties of curcumin: considerations for its clinical development as a cancer chemopreventive and chemotherapeutic agent. Mol Nutr Food Res 2008, 52(Suppl 1): S103-127.

27. Das U, Sharma RK, Dimmock JR: 1,5-Diaryl-3-oxo-1,4-pentadienes: A case for antineoplastics with multiple targets. Current Medicinal Chemistry 2009, 16:2001-2020.

28. Aggarwal BB, Sung B: Pharmacological basis for the role of curcumin in chronic diseases: an age-old spice with modern targets. Trends Pharmacol Sci 2007, 30:85-94.

29. Force T, Krause DS, Van Etten RA: Molecular mechanisms of cardiotoxicity of tyrosine kinase inhibition. Nat Rev Cancer 2007, 7:332-344.

30. Verheul HM, Pinedo HM: Possible molecular mechanisms involved in the toxicity of angiogenesis inhibition. Nat Rev Cancer 2007, 7:475-485.

\section{doi:10.1186/1471-2210-11-4}

Cite this article as: Kudo et al:: Synthesis of 86 species of 1,5-diaryl-3oxo-1,4-pentadienes analogs of curcumin can yield a good lead in vivo. BMC Pharmacology 2011 11:4

\section{Submit your next manuscript to BioMed Central and take full advantage of:}

- Convenient online submission

- Thorough peer review

- No space constraints or color figure charges

- Immediate publication on acceptance

- Inclusion in PubMed, CAS, Scopus and Google Scholar

- Research which is freely available for redistribution 